\title{
The Examination of the Upper Layers of the Seabed by the Means of the Acoustic Methods
}

\author{
E. Kozaczka ${ }^{a, b, c}$, G. Grelowska ${ }^{b}$, W. SzymczaK ${ }^{b}$ And S. KozaczkA ${ }^{b}$ \\ ${ }^{a}$ Gdańsk University of Technology, G. Narutowicza 11/12, 80-233 Gdańsk, Poland \\ ${ }^{b}$ Polish Naval Academy, Śmidowicza 69, 81-103 Gdynia, Poland \\ ${ }^{c}$ University of Technology and Life Sciences in Bydgoszcz, ks. A. Kordeckiego 20, 85-225 Bydgoszcz, Poland

\begin{abstract}
Propagation of acoustic waves in the sea, in particular in shallow sea, depends on several factors. The basic of them are source power and frequency of acoustic waves, spatial distribution inhomogeneous medium in which acoustic wave propagates, and distributions of speed of sound and density of seawater. The coastal conditions, which limit water medium from above (free surface of the sea) and from bottom (the seabed), are no less important. In this paper we will consider the examination of the layer structure of the seabed by the means of the acoustic methods. By the notion of acoustic methods we mean use of phenomena in the field of nonlinear hydroacoustics. Based on this, the parametric sonar was constructed and was used to the seabed sounding. This parametric sonar is commonly called sub-bottom profiler. The selected results of extensive experimental research, that have been performed in waters of the Gulf of Gdańsk in the years 2008-2010, will be presented in this work.
\end{abstract}

PACS: 43.30.Ma, 43.30.Pc, 43.25.Jh

\section{Introduction}

The knowledge of acoustic properties of the seabed is far insufficient, even though this is extensively studied in many fundamental reasons for over half a century. The domain of these studies, in view of deep areas of interest, can be divided. Geo-seismic methods have wide application in research the structure of deep sediments of the sea bottom. In these methods are used strong impulses and also broadband excitations (e.g. explosions), and image is formed as a result of reflection from particular layers. The echo signals are carried to surface from bottom and are recorded by a specially constructed receiving antenna. This range of research gives us information about the structure of the seabed in a relatively approximate term, or a low spatial resolution, in particular in relation to upper layer of seabed.

In view of the above, these methods have small practical usefulness to examination the subtle structure of the upper layers of the seabed [1]. For this reason, other research methods (sounding) of seabed are used, too [2-4].

As is well known, bottoms sediments have a very complex structure of inhomogeneous character and their division is not unambiguous and also obvious matter [5].

It is accepted approximation method of division of materials (substances) of bottom, taking into account the size of elementary particles, which form the appropriate parts of the bottom. In this range, the bottom can be divided into the following macroscopically defined types: rocky, gravel, sandy, loamy, silty, etc. Of course, this division is rather arbitrary, because it is very difficult to find a homogeneous type of bottom in the nature. Generally there are their mixtures, whose contents of a percentage (volume) is difficult to determine in view of strongly inhomogeneous distribution. Therefore, classification of the materials of bottom has statistical character of spatial characteristics and sometimes stochastic (outside layers).

The movement phenomenon, so-called bottom ruble, is commonly known. This phenomenon as a result of demersal water currents causes different changes in the physical properties of the bottom layer.

The mentioned factors make that information contained in geological maps are general in nature and considering for the construction seabed are usually general information and concerning for the construction seabed in particular area for specific cases should be currently verified.

\section{Methods of seabed sounding by the means of acoustic parametric sources}

The parametric sources are constructed based on the discovered phenomenon of nonlinear interaction of acoustic collinear beams of high intensity and small difference of frequency between the waves propagating in particular beams.

This phenomenon has been completely described theoretically and experimentally verified in the last century. To the group of researchers, who put a significant contribution in this area, there belong scientists such as Westervelt, Fubini, Burgers, Ckhockhlov, Kuznetsov, and Zabolotskaya, etc. 
The principle of generation of additional acoustic waves in area of interaction of two acoustic beams of high intensity and small difference of frequency (wavelength) $f_{1}=f_{2}+\Delta f, \Delta f \ll f_{1}, f_{2}$, consist in the fact that the nonlinear characteristics of the medium, in which the wave propagates, cause that the wave of multiples frequency of the fundamental wave is formed in some distance from the source. This wave is called primary wave of their sum and difference.

The most useful wave, in the case of marine research, is the difference frequency wave, also called differential wave. It has special properties, particularly useful to research of the seabed, because it has a narrow directional characteristic, similar to width of the directional characteristics of the primary wave.

Because the source of wave is a certain volume (the end fire type), but not surface, this wave has no so-called side lobes, which is extremely important for the results of the sounding.

Furthermore, the longitudinal resolution, which is connected with the length of formation of impulse of the primary wave is approximately the same.

These basic characteristics of parametric beam, in particular, its low frequency, allow to use these waves successfully to the seabed sounding, because we should say that the wave dissipation by bottom sediments is about thousand times greater than the dissipation in the water.

The disadvantage of parametric generation of acoustic waves is low efficiency of the process, which may be about a few percent.

Therefore, high spatial resolution (longitudinal and angular) and low frequency is a special attribute of the parametric source, and in particular of research of seabed.

\section{Marine examinations - used tools and methods}

In the examination of the seabed there was used a small mobile measurement platform on which measuring-recording apparatus were installed, in this sub-bottom profiler SES-2000 Standard as the most important device.

Measuring echosounder works in the range of primary frequency $f_{1} \approx 100 \mathrm{kHz}$, but differential frequency can change in the range from $4 \mathrm{kHz}$ to $16 \mathrm{kHz}$.

The block diagram of measuring system is shown in Fig. 1 and the diagram of navigation system is shown in Fig. 2.

An important element of the measuring system is system of electronic stabilization of platform motion and thus of the measuring antenna.

In actual conditions it is extremely difficult to keep a constant distance from sea bottom, as well as to keep the condition of perpendicular incidence of the acoustic wave on the seabed. The motion of measuring platform is a result of interaction of dynamic sea surface with the platform, whose results are: pitch, roll, heave, etc.

The task of the motion control system is electronic elimination the influence of these interactions on the ex-

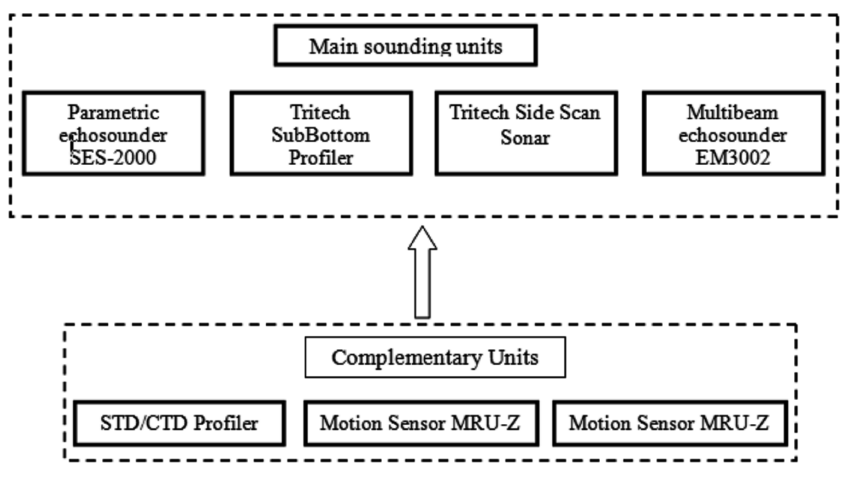

Fig. 1. The diagram of active sounding system for the examination of the seabed.

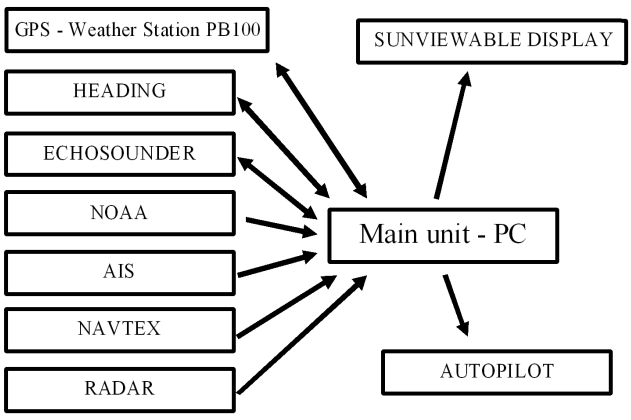

Fig. 2. The diagram of navigation system for the examination of the seabed.

tent that the acoustic beam always fall at the same angle to the bottom (close to zero).

The second important task of measuring system is finding a correlation, and more precisely respectively current (temporary) physical location of the measuring platform in the coordinate system $(\varphi, \lambda)$ with location on the marine map. This is realized by using a precise electronic navigation GPS, DGPS EGNOS (WAAS), which allows for the temporary location of the measuring point in the range 1-3 $\mathrm{m}$, which for this type of measurements is satisfactory.

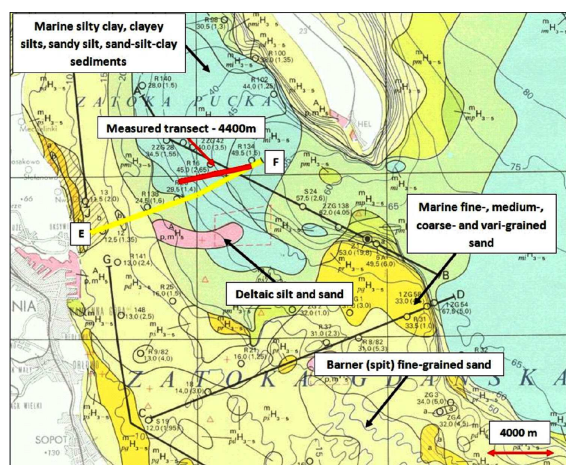

Fig. 3. Geological map with plotted measured transect (thick dark line). 
We can proceed to measurements in the sea after each calibration of the measuring system.

\section{The results of the examinations}

The extensive examinations in waters of the Gulf of Gdańsk, as well as outside, were performed based on previously presented apparatus. The paper presents the results obtained by sounding along the transect (way) as shown in the map (Fig. 3).

\section{Discussion of the examinations results and conclusions}

The examination of the upper structure of the seabed includes the following tasks:

- definition of the layered structure of the sea bottom based on obtained acoustics images,

- possibility of using objects of historical-archaeological character,

— detection buried objects of military-terroristic character,

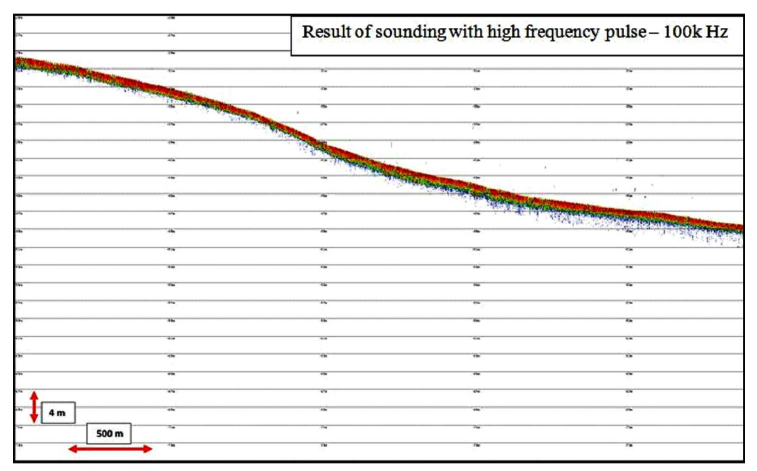

Fig. 4. The image of the sea bottom obtained using classical echosounder.

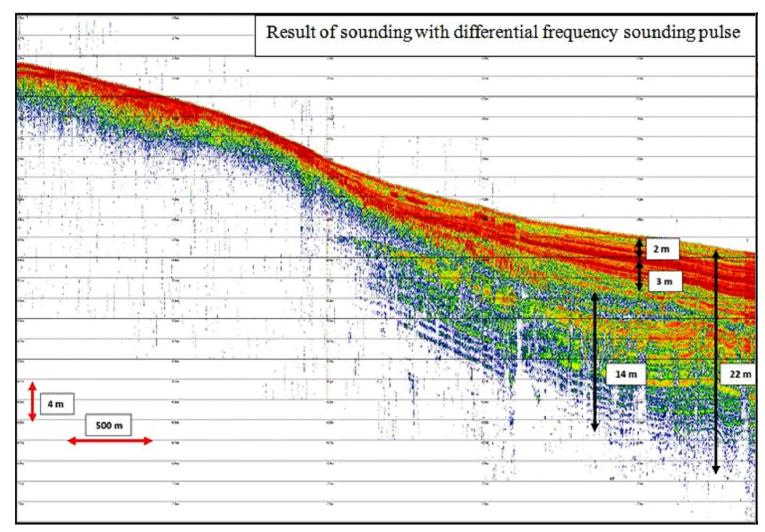

Fig. 5. The image of the sea bottom obtained using parametric echosounder (the same transect as in Fig. 5).

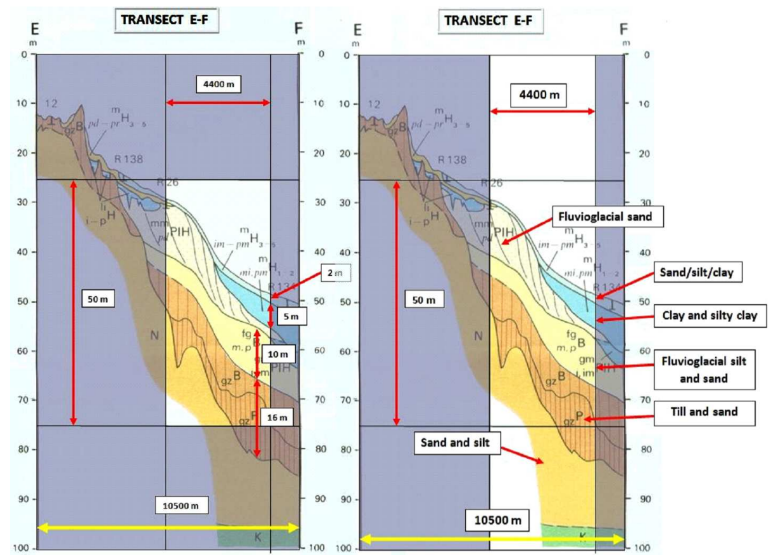

Fig. 6. The geological cross section of the bottom in the investigated area.

- detection and monitoring of an industrial character (pipeline, platforms) etc.,

- monitoring of motion and changes of the layered structure.

These basic tasks can be successfully realized with using acoustic methods. However, only observations of the seabed layering do not add a full knowledge of the structure of the seabed, in particular about physical properties of these layers. Existing and developing method of identifying the structure of the seabed on the basis of the image of sounding are far ambiguous and in this case there must be developed both a theoretical method, mainly based on methods of image recognition, as well as experimental methods.

We should also make use from the observations connected with direct inspection of seabed made by divers (optical observations) and with sampling from the sea bottom surface. In addition, we should take advantage of possibilities of comparisons between structure of the cores taken in selected water medium and search of correlation with the acoustic image.

In the case of performed examinations in waters of the Gulf of Gdańsk, comparison of acoustic images (Figs. 4, 5) with a geological map (Fig. 6) gives satisfactory results. However we should be conscious of the limitations connected with comparison of these results.

The geological maps rarely reflect the subtle structure of the bottom or objects buried into the sea bottom, perhaps with exception of large wrecks or dumps.

\section{References}

[1] G. Grelowska, E. Kozaczka, Nonlinear Properties of Sea Water, AMW, Gdynia 1996, p. 62.

[2] E. Kozaczka, G. Grelowska, S. Kozaczka, Acta Phys. Pol. A 118, 91 (2010). 
[3] Innomar Technologie GmbH, SES-2000 Narrow-beam parametric sub-bottom profilers, Innomar Technologie, Rostock 2008, p. 106.

[4] W. Szymczak, in: 1st EAA - EuroRegio 2010 Congress on Sound and Vibration with Summer School for Young Researchers, Ljubljana, 2010, CD-ROM - 068 [pdf].
[5] G. Grelowska, E. Kozaczka, Acta Phys. Pol. A 118, 66 (2010). 